\title{
CASE OF INTEREST
}

\section{Penetrating Stab Wound of the Heart}

Mr. P. Aged 28 years

THIS civilian patient was admitted to Cambridge Military Hospital on 5 April 1969 at $0848 \mathrm{hrs}$. He had been stabbed in the chest with a potato knife at approximately $0740 \mathrm{hrs}$ that morning during a domestic crisis.

On admission he was semi-conscious, shocked, cyanosed, with congested neck veins, poor peripheral pulse (rate $140^{\circ} \mathrm{min}$ ) and shallow rapid respirations. The blood pressure was unrecordable. The heart sounds were difficult to hear and there was a stab wound below the left nipple. He was diagnosed as having cardiac tamponade.

His arrival at the hospital coincided with that of the duty surgeon Mr. P.J.M.W. who had been called to see another patient and he was taken immediately to theatre. He was transfused with dextran followed by fresh blood as soon as it became available. A left thoracotomy between 6 th and 7 th ribs demonstrated a tense haemopericardium which was incised with removal of a large amount of clot revealing a puncture wound in the anterior wall of the right ventricle. The patient's condition improved after removal of the clot which was followed by a brisk haemorrhage of about 1.5 litres. The ventricle was repaired with interrupted and the pericardium with continuous catgut.

His postoperative course was somewhat stormy, complicated by the fact that he was a drug addict. In the immediate postoperative period he became febrile $\left(\mathrm{T} 102-103^{\circ} \mathrm{F}\right)$ with marked tachycardia (120-140 per minute) and developed heart failure and with it evidence of tricuspid incompetence in the form of marked " $v$ " waves in the neck with systolic pulsation in an easily palpable liver.

In spite of antibiotics and control of his failure, tachycardia 120 per minute persisted for a period. Eventually the rate dropped to $90-100$ per minute. A left parasternal heave with a grade 4 blowing pansystolic murmur at the apex, conducted to the axilla, and at the left sternal border, a grade 3 ejection systolic murmur, became apparent.

There was no significant cardiac enlargement on chest X-ray and e.c.g. was normal. Two weeks after operation his chest wound ruptured and required resuture and one week later he developed right basal pneumonia.

Eventually his symptoms cleared up and he regained his weight loss ( 3 stone) and he only complained of periodic undue fatugue. Bacterial endocardititis was considered at one time but culture was negative and he did not become anaemic, E.S.R. remained normal and clubbing did not develop.

At a later period cardiac catheterisation and angiography demonstrated gross mitral incompetence with considerable reflux into the pulmonary vein. There was no evidence of a ventricular septal defect and right auricular pressure was low with no evidence of tricuspid regurgitation or pulmonary hypertension.

It was presumed the knife had passed through the apex of the right ventricle, and the intraventricular septum and severed some of the chordae of the mitral valve or alternatively had damaged the mitral papillary muscle.

The clinical picture in the postoperative period which suggested tricuspid incompetance was probably due to acute pulmonary hypertension caused by the sudden mitral incompetence. The absence of a residual septal defect is understandable in a stab 
wound. It was thought that surgery would be required later but this has not been necessary as yet.

\section{Comment}

In this country stab wounds of the heart are comparatively rare but several large series have been reported in America.

Injury to the heart should be suspected when there is a wound in or near the chest. Death may occur from blood loss when the wound is open, and haemothorax with respiratory embarrassment may be a feature of this situation. The pericardium may however be sealed by clot with the development of haemopericardium with its classical clinical features. When the chest wound is small the condition may not be diagnosed, with the likelihood of a fatal outcome. Aspiration may be deceptively negative. Haemopericardium or tamponade is recognized by cyanosis with clear airway, shock, distended neck veins, quiet heart sounds, a slow weak pulse and low blood pressure. It may only appear when hypovolaemia is corrected by intravenous therapy.

The management of penetrating wounds of the heart is contraversial some authorities maintaining that paracentesis may be sufficient, thoracotomy being necessary only when no improvement occurs after that procedure. Others maintain that thoracotomy allows full and accurate assessment of the situation. Ballance (1920) in his Bradshaw Lecture in 1919 stated that paracentesis pericardii was a 'leap in the dark' and should be banished from surgical practice.

The immediate treatment is to combat shock by oxygen, intravenous dextran and blood, and in acute tamponade pericardial aspiration may be life saving as an acute emergency measure. However today when advanced cardiac surgery and modern anaesthetic techniques are available we feel that immediate thoracotomy is probably desirable when a penetrating wound of the heart has been diagnosed. It should probably be carried out at the first hospital in which the patient is seen as time precludes transfer to a specialized unit. Certainly this was a life saving procedure in the patient described. In the postoperative period heart failture, bacterial endocarditis and pneumonia must be kept in mind as possible complications.

18 May 1972.

Ballance, Sir C. (1920). Lancet i, 73.
Colonel J. F. WebB

Mr. P. J. M. Wright 